\title{
BAG Family Molecular Chaperone Regulator 4
}

National Cancer Institute

\section{Source}

National Cancer Institute. BAG Family Molecular Chaperone Regulator 4. NCI Thesaurus. Code C26141.

BAG family molecular chaperone regulator 4 (457 aa, $50 \mathrm{kDa}$ ) is encoded by the human BAG4 gene. This protein is involved in the regulation of apoptosis through the suppression of tumor necrosis factor receptor signaling. 